\title{
ANALYSIS OF SURFACE MYOELECTRIC SIGNALS BY LINEAR PREDICTION METHOD
}

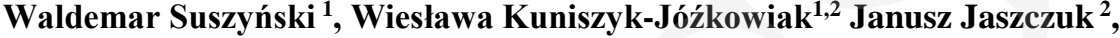 \\ Ireneusz Codello ${ }^{1}$, Rafał Stęgierski ${ }^{1}$, Karol Kuczyński ${ }^{1}$ \\ ${ }^{1}$ Institute of Computer Science, \\ Maria Curie Sklodowska University in Lublin \\ ${ }^{2}$ Faculty of Physical Education and Sport in Biała Podlaska \\ Józef Piłsudski University of Physical Education in Warsaw,
}

KEY WORDS: EMG, linear prediction, Fourier analysis, frequency spectrum.

\begin{abstract}
The article presents a proposal to use linear prediction method for a quick analysis of surface myoelectric (EMG) signals. The spectra obtained with the linear prediction (LP) and Fourier methods were compared. The LP method allows for a precise determination of the location and amplitude of the spectrum maximum and observation of changes in muscle tension and contraction phases. EMG spectra of brachial biceps during flexion and extension of the forearm by four adults were analyzed. The optimal width of the time window for the averaging of motor unit action potentials that allows for the observation of changes during contraction was established. It has been found that maximum spectrum during flexion has a significantly higher frequency and amplitude than during the extension of the forearm..
\end{abstract}

\section{INTRODUCTION}

The human movement is a result of skeletal muscle contractions under the influence of coordinated nerve impulses coming from the central nervous system. The primary control element of muscle contraction is known as motor unit (MU). It consists of many muscle fibers, and under the influence of neurons excitation motor unit action potentials (MUAPs) are generated. Studies on MUAPs are performed with the use of needle electrodes in order to learn about the motor unit structure and diagnose neuromuscular diseases [12-14, 17 19]. Surface EMG signals are the results of summation of many individual MUAPs in time and space. As research studies conducted so far have shown, they contain information about the proper functioning of the muscle, fatigue, strength and processes taking place during the contraction [11]. Surface EMG studies have diagnostic significance in medical problems of the musculoskeletal system, in the evaluation of load and muscle fatigue [9] and in sports medicine. They are also used in the studies of prosthetic control $[8,12]$, and even for speech recognition [1]. In the last decade, there has been a growing interest in the structure of these signals, especially their frequency spectrum. This research typically focuses on the establishment of the mean or median of the Fourier spectrum [5-6]. In recent years there has been considerable interest in the analysis of the potential use of the 
wavelet transform for the analysis of these signals $[7,10,16]$. In the present study, Fourier transform limitations are described and the proposed method of linear prediction is proposed to generalize spectral waveforms. The presented analyses are of a pilot character. The authors intend to make use of parameterized EMG signals to stimulate the movements of a humanoid robot as well as for the recognition of muscle dysfunction in pathological speech articulation and for the application of biofeedback to correct speech defects.

\section{MATERIALS AND METHODS}

Surface myoelectric signals were recorded from the brachial biceps during flexion and extension of the forearm. During the test, Noraxon's second generation of telemetry systems (2400T TeleMyo "G2") was used. Raw EMG signals and the data from the goniometer were recorded. In the pilot study the participants were three adult males (M1, M2 aged 37 and M3 - aged 28) and one woman (K1- aged 63). The surface EMG signals were recorded from the brachial biceps of each subject's right arm. The skin had been abraded and cleaned and bipolar electrodes were placed over the belly of the muscle. Each electrode had a $1 \mathrm{~cm} 2 \mathrm{Ag} / \mathrm{AgCl}$ surface and the inter-electrode distance was $2 \mathrm{~cm}$. The participants were asked to perform five flexion - extension cycles at a fast (approx. $2 \mathrm{~s}$ per cycle) and slow (approx. $5 \mathrm{~s}$ per cycle) rate. The signals were sampled with a frequency of $3000 \mathrm{~Hz}$ and stored in text files. The analysis was conducted using WaveBlaster software elaborated by Codello [2-4]. This program enables Fourier and LP analyses in any selected shape and width of the time window.

\section{THEORY}

\subsection{Short-time fourier spectrum}

In the case of stochastic signals, such as EMG, the Short-Time Fourier Transform (STFT) method can be applied. It is based on an analysis of successive time fragments of the signal multiplied by a window function [20]. This procedure for a digital signal can be represented by the following formula:

$$
X(k)=\frac{1}{N} \sum_{n=1}^{N-1} w(n) \cdot x(n) \cdot e^{-1 \frac{2 \pi}{N} k n}
$$

where $X(k)$ - k-th line of the frequency spectrum, $x(n)-n$-th sample of the signal in the time scale, $\mathrm{w}(\mathrm{n})$ - a function describing the shape of the time window, $\mathrm{N}$ - number of samples for a given width of the time window $(\Delta \mathrm{t})$. In the presented analysis a Hann window was used described by the following function:

$$
w(n)=1 / 2\left(1-\cos \left(\frac{2 \pi n}{N}-1\right)\right.
$$

The width of the time window $(\Delta t)$ determines the resolution of the signal frequency $(\Delta \mathrm{f})$ due to the fact that the total number of spectral lines is equal to the number of samples.

$$
\Delta f \approx \frac{1}{\Delta t}
$$


As it follows from the formula (3), the frequency resolution increases proportionally to the width of the window. The averaged spectrum in a large time window has many distinct peaks in the frequency scale, which creates the problem of deciding which ones are the major peaks. So it is appropriate to use linear prediction method that allows for discretionary smoothing of the spectrum, depending on the choice of the prediction order.

\subsection{LP spectrum}

Linear prediction is a mathematical operation where future values of a discrete-time signal are estimated as a linear function of previous samples [16]. The linear prediction method was originally used primarily for the compression and transmission of voice signals (called Linear Predictive Coding - LPC):

$$
\tilde{y}(n)=\sum_{k=1}^{p} a_{k} y(n-k)
$$

$y(n)$ - the value of the n-th predicted sample y (n) - the value of the n-th sample of actual sample, P-prediction order, $a_{k}$ - prediction coefficients.

The difference between the predicted and the original sample is called the prediction error $\mathrm{e}(\mathrm{n})$ :

$$
e(n)=\tilde{y}(n)-y(n)
$$

The smaller e(n), more accurate the signal approximation. Linear prediction coefficients can be determined by minimizing the mean square error in the vicinity of any given sample. The LP method permits the determination of the frequency on the basis of the following formula:

$$
X(f)=\frac{A}{1-\sum_{k=1}^{p} a_{k} e^{-2 \pi i k f / f_{p}}}
$$

A - gain parameter

$a_{k}-$ LP coefficients

$\mathrm{P}$ - predictor order

$\mathrm{f}_{\mathrm{p}}$ - sampling frequency $[\mathrm{Hz}]$

\section{RESULTS}

\subsection{LP spectra for different time window widths}

Motor unit action potentials in place where the electrode was applied occur in different time moments. Therefore, time-frequency analysis window should be large enough to obtain an adequate averaging of the MUAPs. In the case of Fourier analysis it results in an increase in the number of spectral lines. To extract a clear peak at which the synchronization of the contractions of individual cells is maximal, it is necessary to use the averaging of the spectra. As a result of predictive analysis, the spectra are automatically averaged in the time windows, which allows for the tracking of the maximum synchronization of action potentials.

Figure 1 shows a comparison of spectra: Fourier and LP of the EMG signal recorded during the contraction and extension of the brachial biceps muscle by an adult male. The 
spectra were made with the use of Hann window with the width of 1024 samples (341.3 $\mathrm{ms})$ and an offset of 512 samples $(170.7 \mathrm{~ms})$. The prediction order was 30 . It can be seen that with the same width of the window, the LP spectrum shows distinct groupings in specific frequency ranges. Fourier spectra are very fragmented and difficult to clearly interpret without additional mechanisms of averaging.

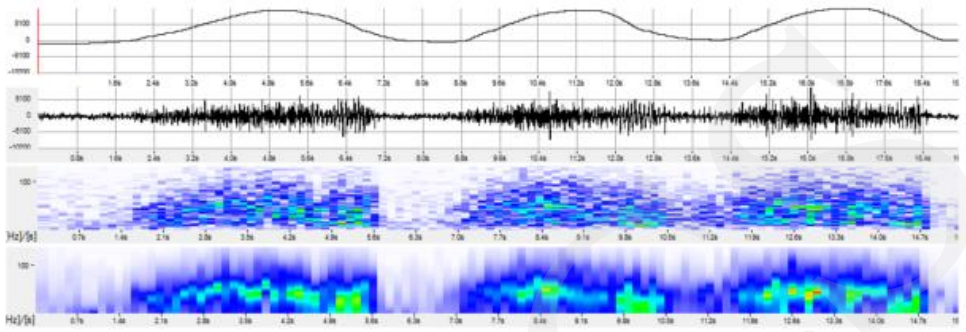

Figure 1 Oscillogram, STFT and LP spectrogram of electromyographic signals recorded during the flexion and extension of the forearm from the brachial biceps. Sequentially from the top of the figure: the indications goniometer, EMG signal waveform, STFT and LP spectrograms.

An important parameter of the frequency analysis is the width of the time window. The selection of this parameter does not only determine the accuracy of the analysis itself, but is also associated with averaging of synchronous potentials derived from individual sarcomeres, significant from the point of muscle functioning. Figure 2 shows the prediction spectrum of brachial biceps EMG signal obtained using the time window widths of $512(170.7 \mathrm{~ms}) 1024$ (341.3 ms) and 2048 (782.7 ms). The signals were recorded during a five cycle flexion/extension of the forearm by a man (M1).

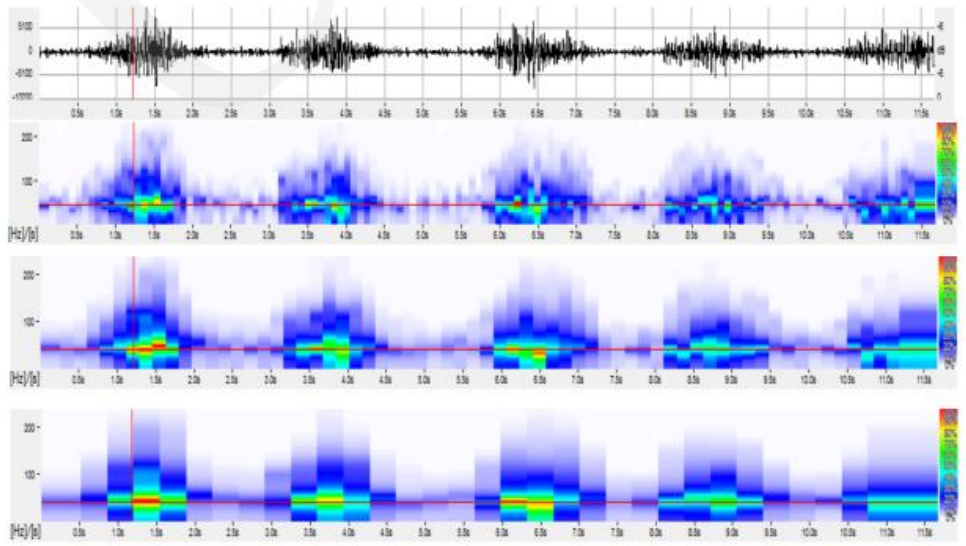

Figure 2 The oscillogram and LP spectrograms obtained using time windows: 512, 1024 and 2048 samples of the brachial biceps EMG signal.

Figure 3 shows a comparison of the frequency characteristics obtained by STFT (Short Time Fourier Transform) and LP method in a chosen time window. As shown, the Fourier course, which contains multiple local maxima, has been smoothed, which allowed for the distinguishing of the main peak. 

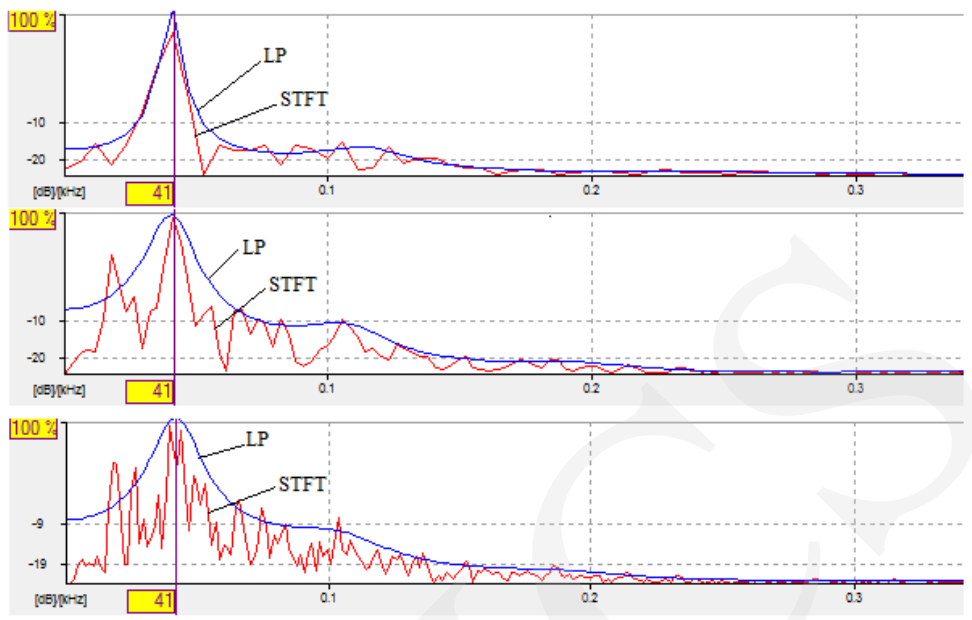

Figure 3 Comparison of STFT (red line) and LP (blue line) frequency characteristics of the biceps EMG signal in the time windows indicated by a pointer in Figure 2. The widths (from top): 512, 1024 and 2048 samples.

The maximum marked in the diagram corresponds to a frequency of $41 \mathrm{~Hz}$. As it can be seen, a maximum frequency location found by the LP spectrum with the use of windows of different widths does not change.

This method offers therefore the possibility of observing the location and the maximum height in different moments of muscle contraction. To date, no research reports the relation of the location of the spectrum to mechanical parameters of the muscles. According to the hypothesis of the authors of the present work, the location can be specific to the type of muscle, its condition and the contraction phase.

The analysis of the spectra obtained in this study indicates that the maximum observed in the extension phase of the muscle is frequently shifted towards lower frequencies, or has a much lower amplitude. The measurements and statistical analysis of the spectral maximum frequencies and amplitudes are presented below.

\subsection{Comparison of the spectral maximums during muscle contraction and extension}

Figure 4 shows a comparison of the spectra in the final phase of contraction and the initial phase of relaxation at the same angle between the arm and forearm. These moments are marked with cursors set on goniometric curves. The top of the illustration shows an EMG signal waveform. The signal was registered in an adult woman. 


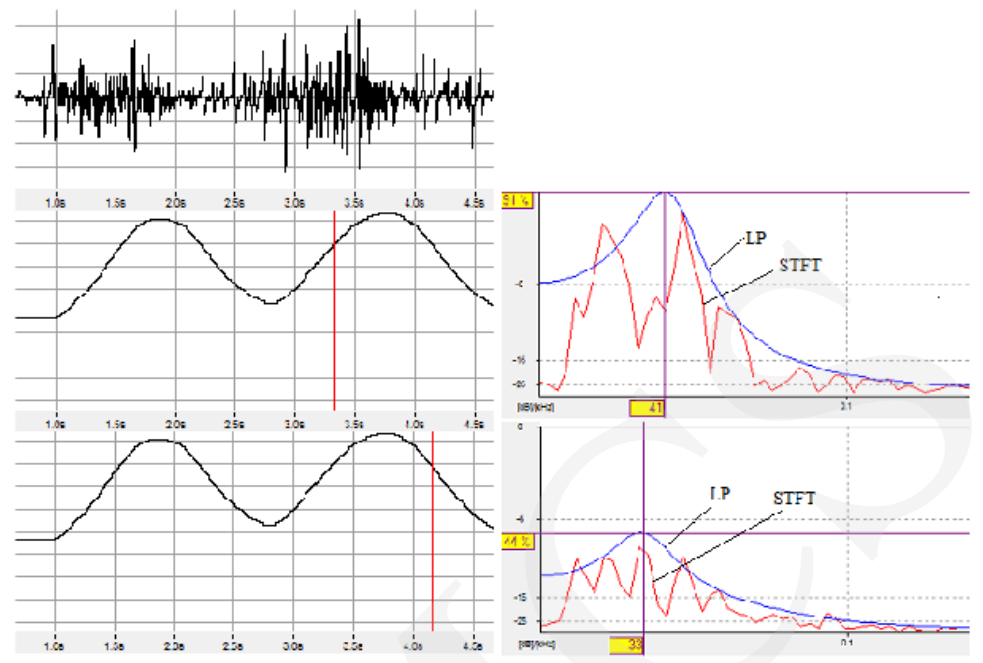

Figure 4 Comparison of the location and the maximum amplitude of the spectrum in the time window at the end of contraction and the beginning of extraction of the biceps muscle of a woman. From the top left: the waveform and two windows on goniometer chart, on the right appropriate STFT (red lines) and LP (blue lines) spectrum

As you can see in Figure 4, the spectrum maximum in the flexion phase is observed at a frequency of $41 \mathrm{~Hz}$, whereas in the extension phase at $33 \mathrm{~Hz}$. The maximum in the contraction phase is moreover steeper and of a higher amplitude. Statistical analysis confirms these observations. The measurements of the peak frequency and maximum amplitude for all spectra at the same angle between arm and forearm in the flexion and extension phases were performed. The histograms are presented in Figures 5 and 6 respectively. 


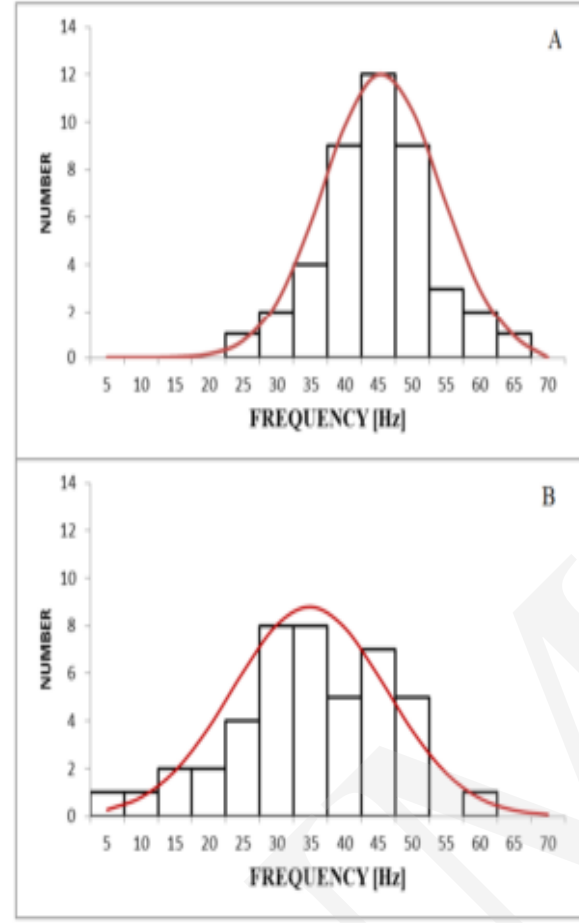

Figure 5 Statistical distributions of the EMG signals LP spectra peak frequencies in the flexion (A) and extension (B) of the forearm.

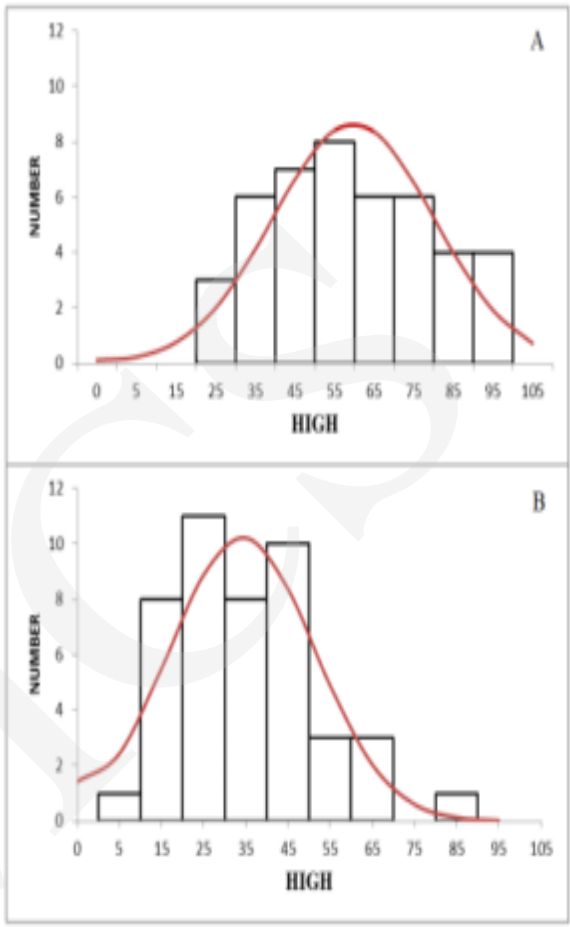

Figure 6 Statistical distributions of LP spectra maximum amplitudes of the EMG signals during flexion (A) and extension (B) of the forearm.

The measurement data were compared with the application of a t-Student test. The peak frequency in the flexion and extension phases are significantly different on the level $\mathrm{p}<0.0005\left(\mathrm{t}_{\text {experimental }}=7.61>\mathrm{t}_{\text {critical }}(40)=3.55\right)$. The difference between the maximum amplitude means was also significant on the level $\mathrm{p}<0.0005\left(\mathrm{t}_{\text {experimental }}=9.74>\right.$ $\left.\mathrm{t}_{\text {critical }}(40)=3.55\right)$.

\section{CONCLUSIONS}

The analysis of the EMG signals is of great significance to the evaluation of the status and functioning of the human muscular-skeletal system. The limitations of the low-latency Fourier Transform (Short-Time Fourier Transform), commonly used in studies of the signals, encourage one to look for better methods. What is interesting from the point of view of diagnostic and rehabilitation activities, such as stimulation with the use of external stimuli, is the averaged spectra from which you can determine the peak frequency of the power spectrum.

The paper proposes a method of linear prediction (LP), which allows for smoothing of the spectrum and for accurate determination of the maximum. The examples of the signal biceps muscle analyses presented in the paper indicate that this method allows for 
a precise determination of the dominant frequency (peak frequency) regardless of the width of the time window. The determination of the location of the spectrum's peak frequency and its amplitude can be used to assess the state of the muscle and is also important for the selection of rehabilitation frequency signals. The authors plan to use the described method to observe the synchronization of muscle activation in fluent and disfluent speech articulation, to observe the propagation of excitation along the muscle fibers and to apply EMG signals to control the humanoid robot's movements.

\section{LITERATURE}

[1] Betts B J, Binsted K, Jorgensen Ch. Small-vocabulary speech recognition using surface electromyography. Interacting with Computers 2006; 18: 1242-59.

[2] Codello I, Kuniszyk-Jóźkowiak W. Digital signals analysis with the LPC method. Annales UMCS Informatica 2006; A15: 315-21.

[3] Codello I, Kuniszyk-Jóźkowiak W.'Wave Blaster' - A comprehensive tool for speech analysis and its application for vowel recognition using wavelet continuous transform with bark scale. In: Proc. 56th open Seminar in Acoustics 2009; 141-46.

[4] Codello I, Kuniszyk-Jóźkowiak W, Smołka E, Suszyński W. Speaker Recognition Using Continuous Wavelet Transform with Bark Scales. Polish Journal of Environmental Studies 2009; 18 (3B): 78-82.

[5] Coorevits P, Danneels L, Cambier D, Ramon H, Druyts H, Karlsson J S, De Moor G, Vanderstraeten G. Correlations between short-time Fourier and continuous wavelet transforms in the analysis of localized back and hip muscle fatigue during isometric contractions. Journal of Electromyography and Kinesiology 2008; 18: 637-644.

[6] Fele-Žorž G, Kavšek G, Novak-Antolič Ž, Jager F. A comparison of various linear and non-linear processing techniques to separate EMG records of term and pre-term delivery groups. Med. Biol. Eng. Comp. 2008; 46: 911-22.

[7] Flanders M. Choosing a wavelet for single-trial EMG. Journal of Neuroscience Methods 2002; 116: 165-177.

[8] Manal K, Buchanan T S. A one-parameter neural activation to muscle activation model: estimating isometric joint moments from electromyograms. Journal of Biomechanics 2003; 36: 1197-1203.

[9] Moshou D, Hostens I, Papaioannou, Ramon H. Dynamic muscle fatigue detection using self-organizing maps. Applied Soft Computing 2005; 5: 391-398.

[10] Olmo G, Laterza F, Lo Presti L, Matched wavelet approach in stretching analysis of electrically evoked surface EMG signal. Signal Processing 2000; 80: 671-684.

[11] Farina D, Falla D, Estimation of muscle conduction velocity from two-dimensional surface EMG recordings in dynamic tasks. Biomechanical Signal Processing and Control 2008; 3: 138-144.

[12] Piotrkiewicz M, Hausmanowa-Petrusewicz I, Mierzejewska J. Are motoneurons involved in muscular dystrophy? Clinical Neurophysiology 1999; 110: 1111-22.

[13] Piotrkiewicz M, Filipiuk M, Hausmanowa-Petrusewicz I. MU firing characteristics in human dystrophic muscle. Acta neurobiologiae experimentalis. 1993; 53(1): 31318.

[14] Piotrkiewicz M, Kudina 1, Chen JJJ, Zalewska E, Hausmanowa-Petrusewicz I. Assessment of Human Motoneuron Afterhyperpolarization Duration in Health and Disease. Biocybernetics and Biomedical Engineering 2012; 32 (3): 43-61.

[15] Rabiner LR, Schafer, Digital Processing of Speech Signals. New Jersey; PrenticeHall, Inc. ; 1978. 
[16] von Tscharner V, Goephert B, Nigg BM. Changes in EMG signals for muscle tibialis anterior while running barefoot or with shoes resolved by non-lenarly scaled wavelets. Journal of Biomechanics 2003; 36: 1169-76.

[17] Zalewska E, Hausmanowa-Petrusewicz I. Approximation of motor unit structure from the analysis of motor unit potential. Clinical Neurophysiology 2008; 119: 2501-06.

[18] Zalewska E, Hausmanowa-Petrusewicz I. Effectiveness of motor unit potentials classification using various parameters and indexes. Clinical Neurophysiology 2000; 111: 1380-1387.

[19] Zalewska E, Rowinska-Marcinska K, Gawel M, Hausmanowa-Petrusewicz I. Simulation studies on the motor unit potentials with satellite components in amyotrophic lateral sclerosis and spinal muscle atrophy, Muscle \& Nerve 2012; 45 (4): 514-521.

[20] Zieliński T P. Digital Signal Processing. Warszawa; Publisher Transport and Communications; 2005 (in Polish). 